Article

\title{
Classification of Refrigerant Flow Noise of Air Conditioners Based on Continuous Wavelet Transform and Support Vector Machine
}

\author{
Un-Chang Jeong \\ Department of Automotive Engineering, Honam University, Gwangsan-gu, Gwangju 62399, Korea; \\ ucjeong@honam.ac.kr
}

Received: 12 May 2020; Accepted: 5 June 2020; Published: 7 June 2020

\begin{abstract}
This study proposes a classification method that uses the continuous wavelet transform and the support vector machine approach to classify refrigerant flow noises generated in an air conditioner. The air conditioning noise was identified as an abnormal signal by the use of the firstand second-order moments. The start and end times of refrigerant flow noises were identified by detecting the singularities of the continuous wavelet transform coefficient in the time domain and by means of listening to the measured sounds. Further, the time-frequency characteristics of refrigerant flow noise were analyzed with the continuous wavelet transform. For the support vector machine-based classification of refrigerant flow noise in an air conditioner, the grid search method was used to determine kernel hyperparameters. Five-fold cross validation was employed for the application of the support vector machine to the classification of air conditioner refrigerant noise. In addition, measured sound sources were modified based on classified refrigerant flow noise to compare the classification accuracy of a jury test with the results of the support vector machine.
\end{abstract}

Keywords: refrigerant flow noise; continuous wavelet transform; support vector machine; classification

\section{Introduction}

Home appliances have a significant influence on the living conditions of consumers. The vibration of home appliances can cause interior noise in the home and make people uncomfortable. Consumers need to remove the irritating feeling by reducing such noise and vibration. Although air conditioner noise is low level, there are still a lot of complaints from consumers about the noise, particularly from indoor units of air conditioners, which affects their purchasing decisions. Air conditioner noise is known to be generated mainly due to the refrigerant flowing in air conditioning systems. In an air conditioner, the refrigerant is a medium for conveying heat. For heat transfer, the refrigerant repeats evaporation, compression, condensation and expansion, during which two-phase flow occurs due to nonlinear phase changes of the refrigerant. As a result, the flow pattern varies and a wide frequency range of noise is generated. In general, research on refrigerants has been mainly done from a thermodynamic perspective [1,2]. Han and Jeong classified two-phase flows into some regimes: slug flow, churn flow and annular flow $[3,4]$. The study of air conditioning refrigerant noise mainly focuses on theoretical and experimental identification of noise from pipes.

However, the characteristics of refrigerant flow noise from air conditioners have not been identified, and there has been no method suggested for classification of the noise. Malcom J. Crocker et al. identified the noise sources on a residential split-system air conditioner using sound intensity measurements. However, this was not the refrigerant flow noise, but rather was the inlet and exhaust noise [5]. Jiang et al. conducted an experimental and numerical study on axial flow fan noise of a room air conditioner [6]. Therefore, the study of the identification of air conditioning refrigerant noise is needed. 
To do this, refrigerant noise should first be classified. In general, a jury test, used to classify the noise, or sound quality index through regression method, is widely used [7]. Yoshiharu et al. conducted sound quality evaluation of air conditioner noise based on autocorrelation [8], and Susini et al. characterized the sound quality of air conditioning noise [9]. Shengmei et al. developed a technique for evaluating the sound quality of an air conditioner [10]. However, the results of evaluations using this method can vary according to different listeners and it is difficult to identify when the noises occur. Therefore, refrigerant flow noise needs to be accurately classified for the reduction of such noise. In order to achieve this purpose, the start/end time and frequency characteristics of air conditioner refrigerant flow noise should be investigated and identified to ensure accurate classification of the refrigerant flow noise.

In identifying the start/end time of the flow noise- the characteristics of which vary with time-it is important to find singularities in which changes occur due to the generation of refrigerant flow noise in the signal. Because the refrigerant flow noises are abnormal signals, it is possible to detect the time of occurrence by using the continuous wavelet transform.

The continuous wavelet transform (CWT) is widely used to determine when rapid changes occur in frequency characteristics or when signal singularities appear. Signal singularities are the points in which the stead -state changes to other states. The CWT is also used to detect abnormal signals generated by earthquakes, shock or engine acceleration or deceleration [11-14]. In other words, abnormal signals are detected with large changes in the CWT coefficient at signal singularities.

The frequency characteristics of abnormal signals can be analyzed by using a time-frequency analysis method, such as short-time Fourier transform (STFT) or CWT. STFT has a fixed window size and a low frequency resolution which depends on low- and high-frequency domains. In contrast, the CWT-based time-frequency analysis approach provides better resolution both in time and frequency [15].

Meanwhile, a support vector machine (SVM) is a supervised machine learning model that can classify the data. It can be applied to classifying the noises of refrigerant flow by learning the signals identified by the CWT. The SVM tries to obtain a hyperplane for generalization errors to decrease in the learning process. The SVM defines the objective function for learning by using a margin to find the optimal boundary out of a number of hyperplanes. This is to find the hyperplane that maximizes this margin. If it is impossible to classify data linearly, the data can be mapped into a high dimensional space for separation by introducing a slack variable or exploiting a kernel $[16,17]$. When the kernel-based SVM is used, an appropriate hyperparameter value should be selected for the kernel function. In general, a grid search method is used to determine the hyperparameter value. This method tries to find optimal parameter values within a fixed range. The classification performance of the SVM can be enhanced once an appropriate kernel hyperparameter is determined by the grid search method and applied to the SVM.

Noise classification can be performed using a jury test, but noise can be classified using a hyperplane, without the need for experts, through an SVM. It also has the advantage of being able to consistently evaluate air conditioning refrigerant noise. In addition, if refrigerant noise occurs, it can be identified using CWT, enabling noise control through refrigerant control.

To evaluate the performance of SVM with regards to other refrigerant flow noises, noises are modified and compared by jury test. Modified sound sources are produced through amplification/reduction or frequency modulation of the frequency band that characterizes the refrigerant flow noises.

This study proposed a method for classifying air conditioner refrigerant flow noise based on the SVM and the CWT. To this end, the first- and second-order moments were used to determine if the flow noises were abnormal signals in Section 3. In Section 4, the occurrence time of refrigerant flow noise were identified by finding the singular points of the CWT coefficient and locating the start and end points by means of listening to sounds. Further, the CWT was used to analyze the time-frequency characteristics in the identified start/end time of refrigerant flow noise. An appropriate kernel 
hyperparameter was obtained by a grid search method, and the SVM-based classification of refrigerant flow noise was performed by means of 5-fold cross validation in Section 5. In addition, an enhanced method for classification of refrigerant flow noise was presented with the kernel feature vectors of the SVM in the analyzed frequency characteristics. Finally, measured sound sources are produced based on classified refrigerant flow noises to compare the classification accuracy through a jury test and SVM in Section 6.

\section{Theory}

\subsection{Continuous Wavelet Transform}

The continuous wavelet transform defined by Morlet and Grossman is as follows [18]:

$$
W_{f}^{\Psi}(a, b)=\frac{1}{\sqrt{a}} \int_{-\infty}^{\infty} x(t) \Psi\left(\frac{t-b}{a}\right) d t
$$

where $x(t)$ denotes the signal, $a$ is a scale parameter, $b$ is a shift parameter and $W_{f}^{\Psi}(a, b)$ is the wavelet coefficient. All the parameters and functions are defined in the domain of real numbers.

The outstanding characteristic of the CWT, which can be known from this equation, is to expand or reduce the predefined wavelet $\Psi(t)$ using the scale factor $a$ and shift the wavelet using the shift factor $b$ which indicates a location in the time domain.

As for the CWT, the wavelet is gradually dilated with increasing scale parameter $a$ and reduced as the scale parameter gets smaller. Therefore, the scale parameter is small at high frequencies and large at low frequencies. These properties of the CWT make it possible to plot signal characteristics on a plane of two axes, time and frequency. Signals can become easy to interpret when their wavelet coefficients are plotted on the time-frequency plane, and the time-frequency interpretation is useful for the interpretation of abnormal signals.

\subsection{Support Vector Machine}

A support vector machine (SVM) is a kind of supervised machine learning technique based on statistical learning theory, used for classification and regression analysis. The SVM constructs a set of hyperplanes, a concept of decision planes that define decision boundaries. For separable classification problems, the SVM algorithm finds an optimal separating hyperplane. The form of equation defining the decision surface that classifies is as follows:

$$
w \cdot x+b=0
$$

which implies

$$
y_{i}(\boldsymbol{w} \cdot x+b) \geq 1, \quad i=1,2, \cdots, N
$$

where $y_{\mathrm{i}}$ is the sign of $i$-th data point, $w$ denotes the normal vector of the hyperplane, $x$ denotes the input data and $b$ is bias [19].

By maximizing the margin of separation subject to this constraint, the SVM tries to find an optimal hyperplane. Here, the margin of separation is the distance between the hyperplane and the support vector for a given weight vector $w$ and bias $b$.

If it is not possible to classify data linearly, the data can be classified by a kernel SVM. The use of the kernel function allows data to be separated without increasing the computational complexity, despite mapping into a high-dimensional space. The radial basis function (RBF) kernel is the most popular kernel which can be expressed as follows:

$$
Z=e^{-\frac{\left\|x-X_{j}\right\|^{2}}{2 \delta^{2}}}
$$


where $x$ is the input data and $X_{j}$ is the center of the radial basis function. The classification performance of the SVM is influenced by two hyperparameters of the RBF. C is the SVM's penalty hyperparameter, and $\delta$ is the kernel hyperparameter. A grid search is the most common approach for determining the two hyperparameters. The idea behind the grid search method is to find an optimal parameter by selecting discrete values at specified intervals. Basically, this method uses the pairs of $(C, \delta)$ for cross-validation and selects the value of highest cross-validation accuracy. The exponentially increasing value of $(C, \delta)$ is a way to find the optimal appropriate parameter.

In this work, the appropriate values of $C$ and $\delta$ were changed to the form of $2^{n}$ in order to find the appropriate values. Generally, the form of $10^{n}$ is used. However, in the form of $10^{n}$, the values of $\mathrm{C}$ and $\delta$ vary greatly depending on $n$. Hence, using a form of $2^{n}$ was more suitable to get the appropriate values. With the selection of parameters, the classification with SVM is performed to find the parameter where the best classification performance is achieved. In this study, $k$-fold cross validation was used for the SVM-based classification.

In $k$-fold cross validation, the original data are divided into $k$ subsamples. A single subsample remains as the validation data for testing the model. The retained $k-1$ subsamples are used as training data. The cross-validation process is repeated $k$ times until the $k$ samples are used exactly once as the validation data. After that, the results are averaged to produce a single estimation. In this validation method, all subsamples are used once for training and validation, and each of them is used exactly once as the validation data.

\section{Experiment on Refrigerant Flow Noise}

\subsection{Experimental Setup and Schematic Diagram}

A microphone (B\&K Type 4189) and FFT analyzer (Muller-BBM PAK-MK II) were used to measure the refrigerant flow noise generated in an air conditioner. The most common wall-mounted model (WM) was selected as an air conditioner for use in the measurement of refrigerant flow noise. The refrigerant flow noise from the air conditioner was measured in an anechoic room. Figure 1 shows an experimental setup for measuring the refrigerant flow noise. Noise was measured at a distance of one meter and at a height of $0.8 \mathrm{~m}$ (sitting position).
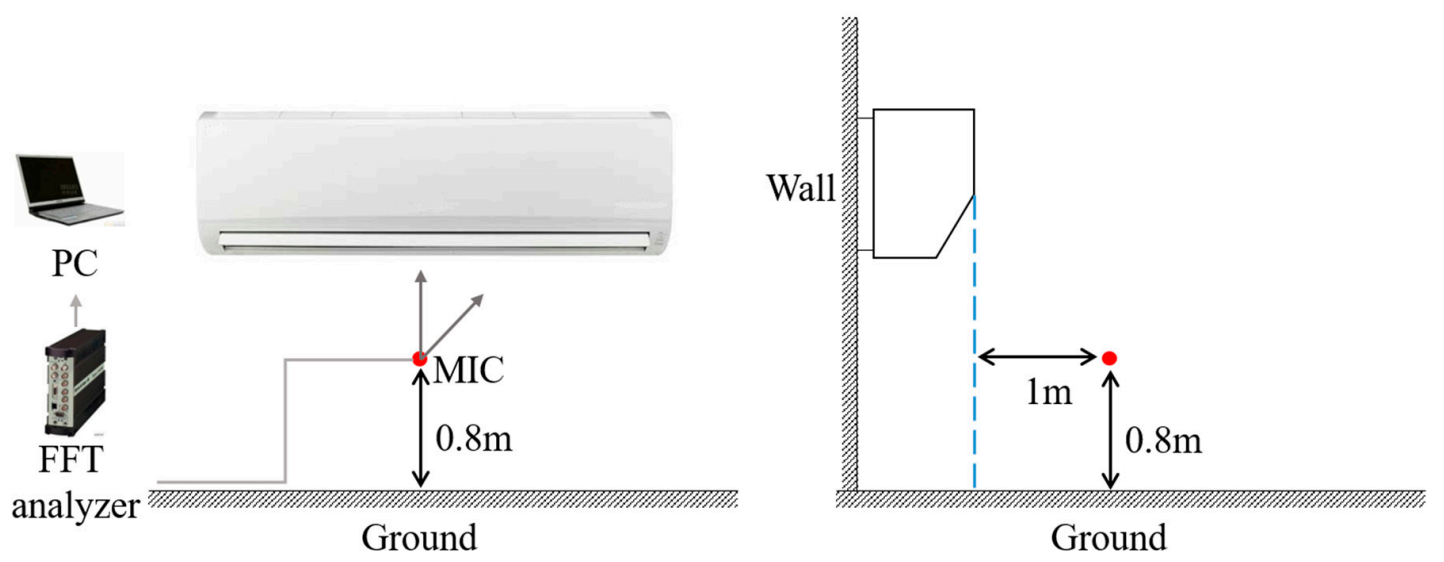

Figure 1. Experimental setup.

The air conditioner operated in defrost mode to generate a wide frequency range of refrigerant flow noise. The system was allowed to operate for $600 \mathrm{~s}$ to produce sufficient refrigerant flow noise.

\subsection{Noise Measurements and Identification of Abnormal Signals}

Figure 2 shows the results from the measurement of the air conditioning noise. In this section, Equations (6) and (7) were used to calculate the first- and second-order moments of the measured refrigerant flow noise and determine whether it was an abnormal signal. Signals are normal when 
there are negligible changes in their first- and second-order moments at a specific time for each sample. Abnormal signals, on the other hand, have the first- and second-order moments varying with time.

$$
\begin{gathered}
\mu=\mathrm{E}[x]=\frac{1}{n} \sum_{i=1}^{n} x_{i} \\
\sigma^{2}=\mathrm{E}\left[(x-\mu)^{2}\right]=\frac{1}{n} \sum_{i=1}^{n}\left(x_{i}-\mu\right)^{2}
\end{gathered}
$$

where $n$ denotes the number of data points in the time window considered and $x_{i}$ represents the $i$-th sound pressure sample.

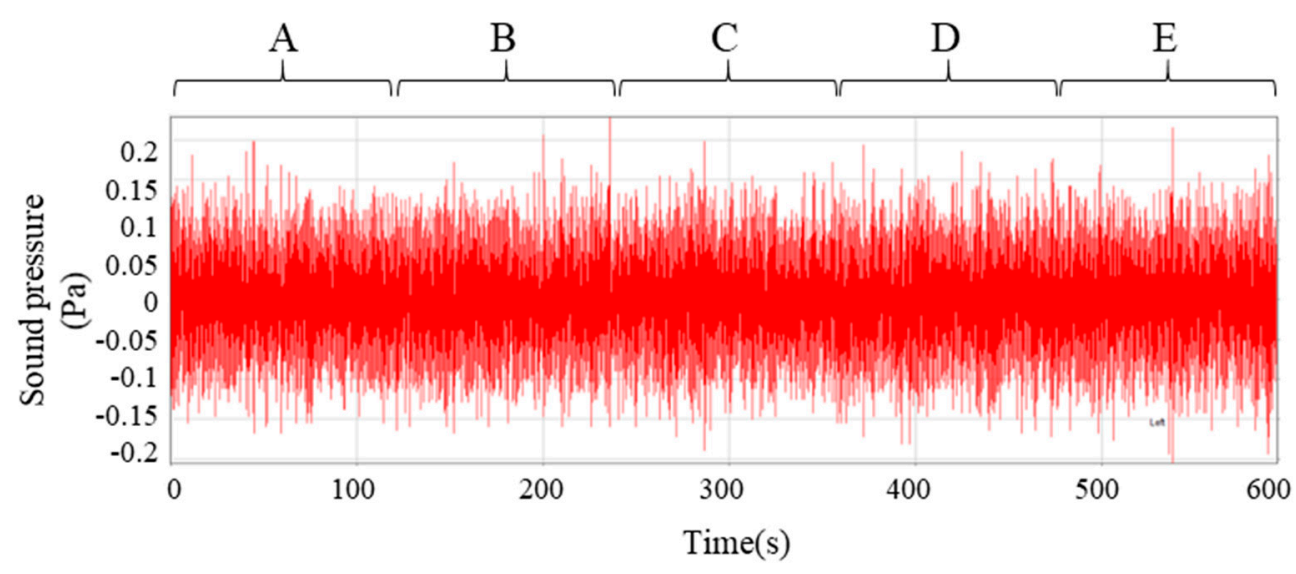

Figure 2. Measurements of refrigerant flow noise.

The measured noise signals were divided into five equal time intervals (0-120, 120-240, 240-360, 360-480 and 480-600 s). No refrigerant noise was heard until 0-120 s, but $120 \mathrm{~s}$ later the refrigerant noise could be heard. Thus, an interval of $120 \mathrm{~s}$ was set to evenly distribute the number of data. Table 1 shows the first- and second-order moments of the noise in each time interval.

Table 1. First- and second-order moments of refrigerant flow noise.

\begin{tabular}{cccccc}
\hline & A & B & C & D & E \\
\hline$\mu$ & $-1.0 \times 10^{-3}$ & $8.0 \times 10^{-3}$ & $3.0 \times 10^{-3}$ & $2.3 \times 10^{-3}$ & $-1.5 \times 10^{-3}$ \\
$\sigma^{2}$ & $5.7 \times 10^{-3}$ & $5.1 \times 10^{-3}$ & $4.1 \times 10^{-3}$ & $5.0 \times 10^{-3}$ & $4.7 \times 10^{-3}$ \\
\hline
\end{tabular}

Both the first- and second-order moments varied with the time interval (Table 1). The air conditioner noise exhibited time-varying non-stationary characteristics. Accordingly, the continuous wavelet transform was employed to identify the start/end time and characteristics of refrigerant flow noise generated.

\section{The Time of Occurrence and Frequency Characteristics Identification}

\subsection{The Identification of the Start/End Time}

In this section, CWT is used to identify the start/end time of refrigerant flow noise. In this study, the Daubechies 4 wavelet, which has been most widely used, was selected. It is commonly used due to its great performance against irregular signals. Using the Daubechies 4 wavelet, wavelet coefficients can be estimated as follows (Figure 3): 


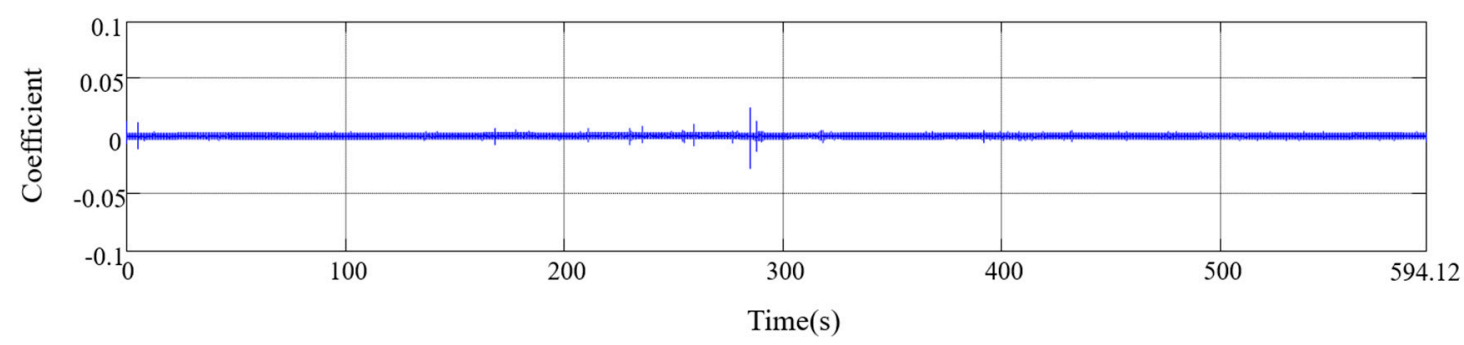

(a)

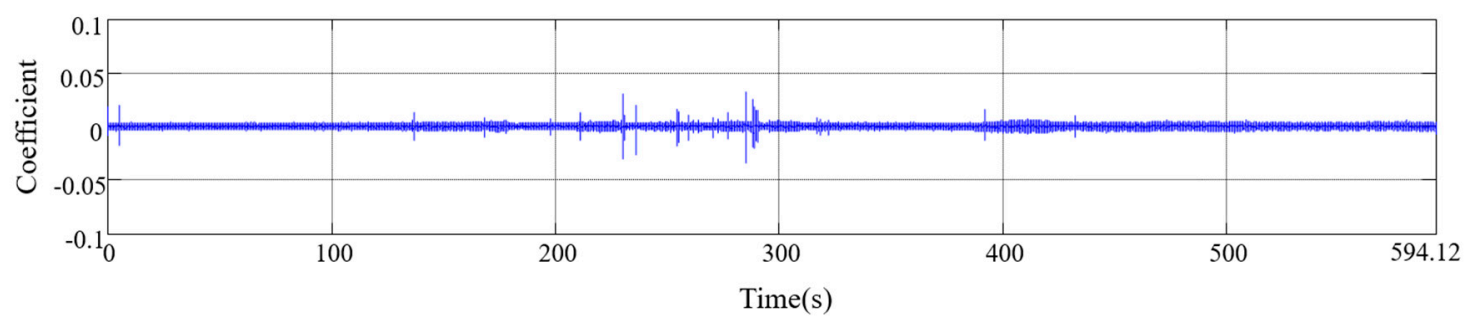

(b)

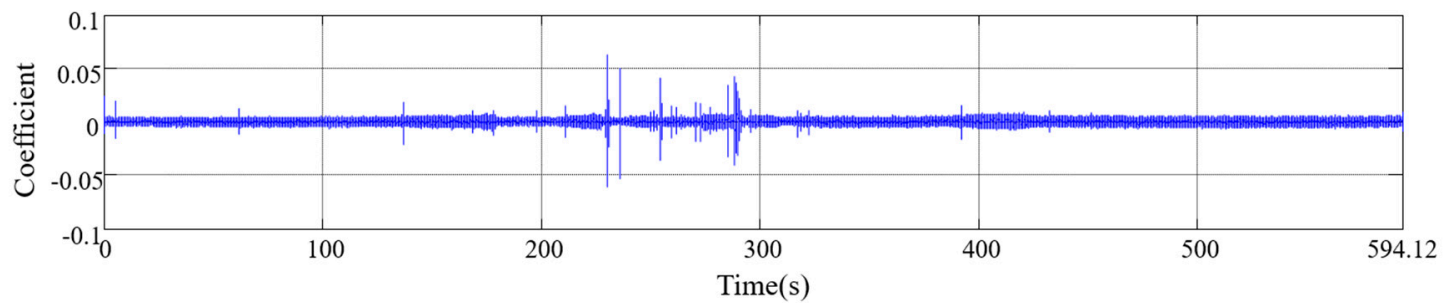

(c)

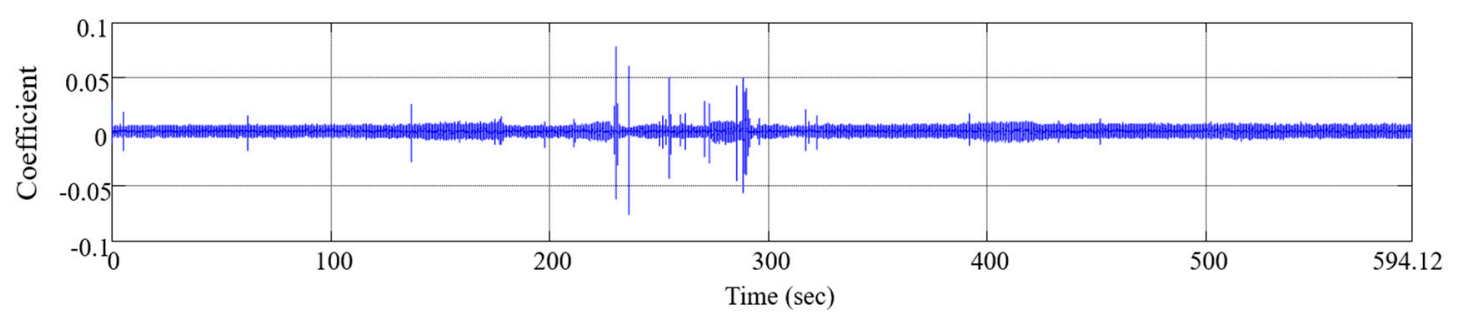

(d)

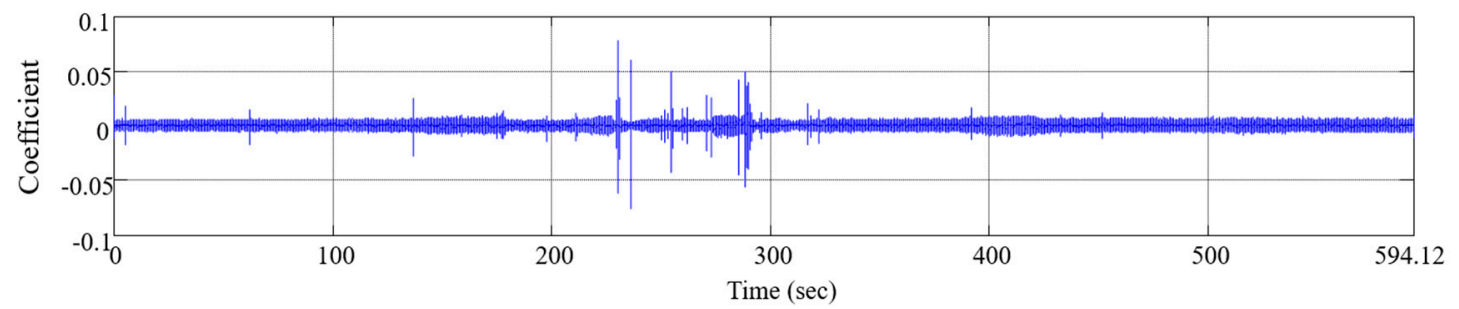

(e)

Figure 3. Continuous Wavelet Coefficient: (a) Scale: 5, (b) Scale: 10, (c) Scale: 15, (d) Scale: 20, (e) Scale: 25 . 
The scale represents $a$ in Equation (1) and can either dilate or compress a signal as the scale changes. High wavelet coefficients refer to a point where signals rapidly change, and this spot is the start or end of the refrigerant flow noise. Figure 4 below shows individual scales' coefficients $(5,10$, 15,20 and 25) for setting the criteria to identify this point:

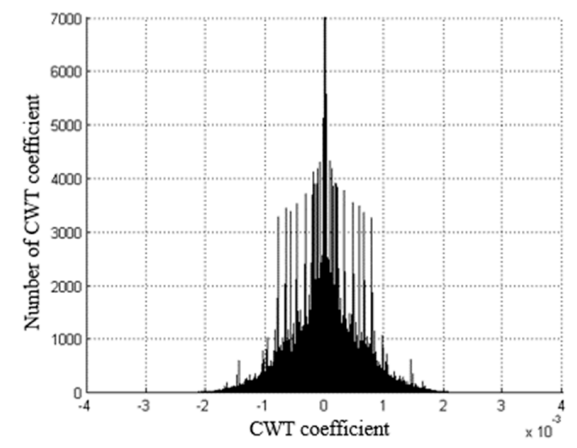

(a)

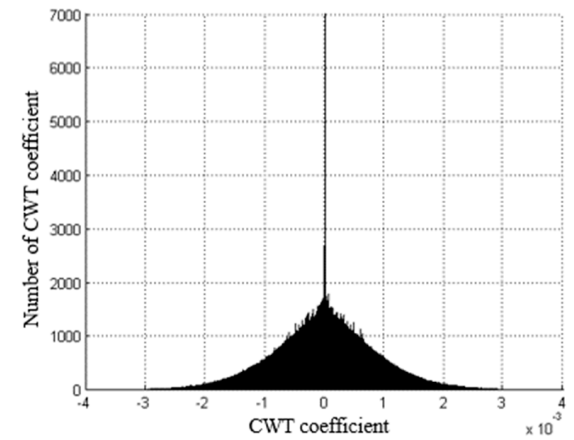

(b)

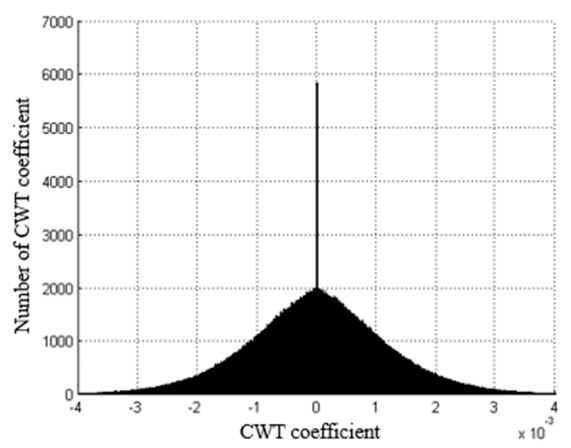

(c)

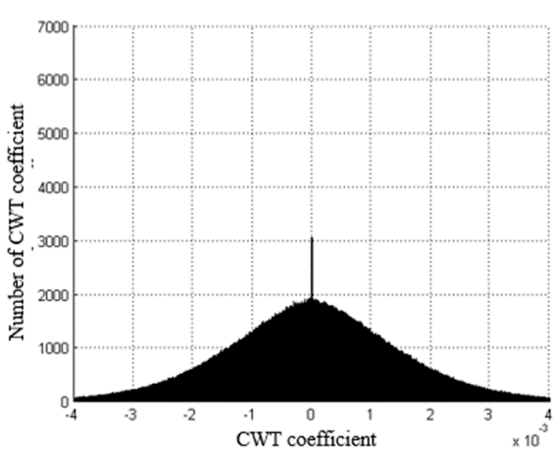

(d)

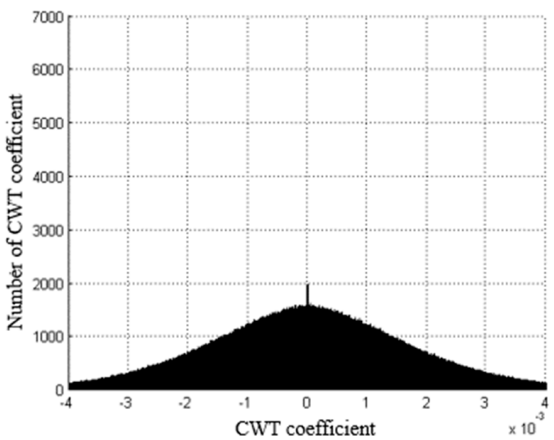

(e)

Figure 4. Distribution of continuous wavelet coefficients: (a) Scale: 5, (b) Scale: 10, (c) Scale: 15, (d) Scale: 20, (e) Scale: 25.

Each scale's average is the same with zero, but different variances were found in distribution curves. The CWT coefficients revealed the highest distribution at zero. If original signals rapidly change, the CWT coefficients' absolute values fluctuate greatly. Hence, each scale's 99.5th percentile of distribution was used to classify a singular point. Table 2 lists the 99.5th percentiles of CWT coefficients by scale. 
Table 2. 99.5th percentile of the CWT coefficient and singular points (s).

\begin{tabular}{|c|c|c|}
\hline Scale & 99.5th Percentile & Singular Point (s) \\
\hline 5 & 0.002 & $5, \mathbf{1 7 0}, 209,228, \mathbf{2 3 2}, 254,260,278,286, \mathbf{3 1 3}, \mathbf{3 1 7}$ \\
\hline 10 & 0.004 & $5, \mathbf{1 4 4}, \mathbf{1 7 0}, \mathbf{1 9 8}, 228, \mathbf{2 3 2}, 254,260,278,286,288,313,317,393,432$ \\
\hline 15 & 0.007 & $5,61, \mathbf{1 4 4}, \mathbf{1 7 0}, \mathbf{1 9 8}, 228, \mathbf{2 3 2}, 254,260,278,286,288,313,317,393,432$ \\
\hline 20 & 0.011 & $5,61, \mathbf{1 4 4}, \mathbf{1 7 0}, \mathbf{1 9 8}, 228, \mathbf{2 3 2}, 254,260,278,286,288,313,317$ \\
\hline 25 & 0.015 & $5,61, \mathbf{1 4 4}, \mathbf{1 7 0}, \mathbf{1 9 8}, 228, \mathbf{2 3 2}, 254,260,278,286,288,313,317$ \\
\hline
\end{tabular}

In Table 2, the most common singular points are 144-170, 198-232 and 313-317 s (see Table 2 for the boldface). A jury test was conducted with 16 experts on air conditioner noise. Each noise interval was heard by experts to determine whether it was slug, churn or annular noise. In this test, three scales were used, and the expert listeners were asked to answer whether they could hear or not, or if they had a neutral opinion (not sure). Then it was identified that they were the starting/ending points of refrigerant flow noises. In addition, different characteristics of refrigerant flow noises were observed in these time intervals through the sound test. According to each time interval of refrigerant flow noise, therefore, 144-170, 198-232 and 313-317 s were categorized as slug flow, churn flow and annular flow, respectively.

For classification of refrigerant flow noise, an SVM was conducted on the refrigerant flow noise and its frequency characteristics in each time interval.

\subsection{The Frequency Characteristics Identification of Refrigerant Flow Noise}

Figure 5 shows the time intervals identified with CWT coefficients and through the jury test. Because it appears that different refrigerant flow noises take place among the time intervals, it is necessary to determine the characteristics through time-frequency analysis. The time-frequency analysis in Figure 6 shows that frequency characteristics varied in three time intervals at $\leq 3000 \mathrm{~Hz}$. As shown in Figure 7, a high level was found at 800-1200 Hz and 2100-2300 Hz in the 1st and 2nd time intervals and at $1056-1356 \mathrm{~Hz}$ and $2085-2385 \mathrm{~Hz}$ in the 3rd time interval.

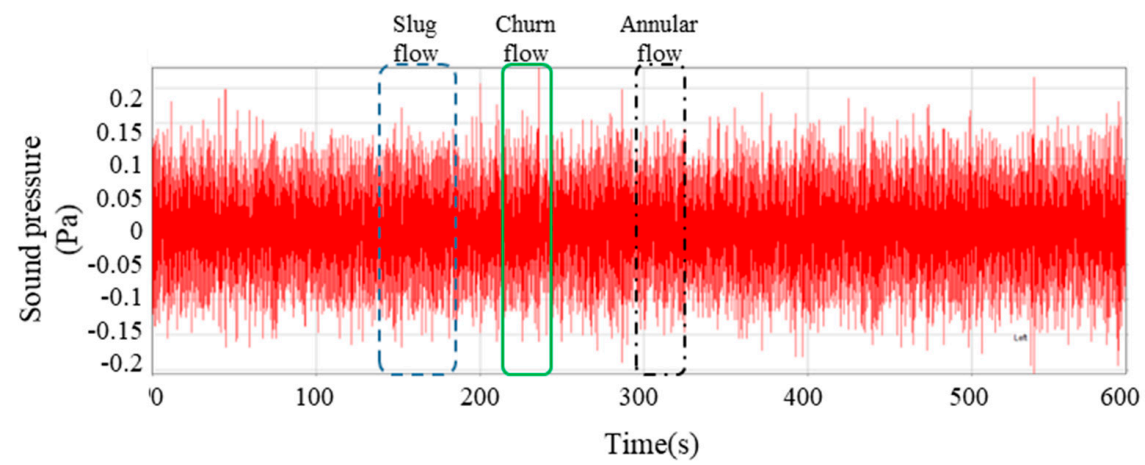

Figure 5. Slug, churn and annular noises of refrigerant flow noise.

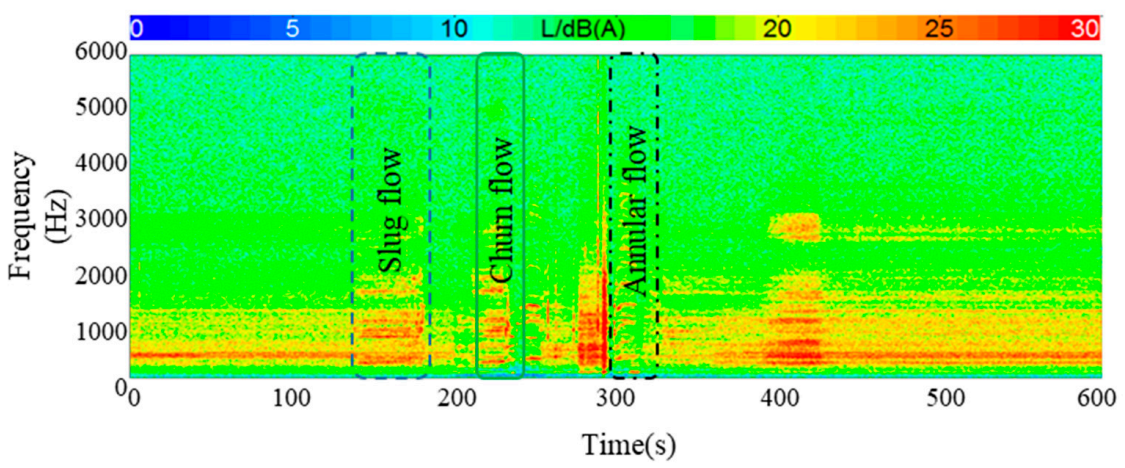

Figure 6. Time-frequency analysis of refrigerant flow noise. 


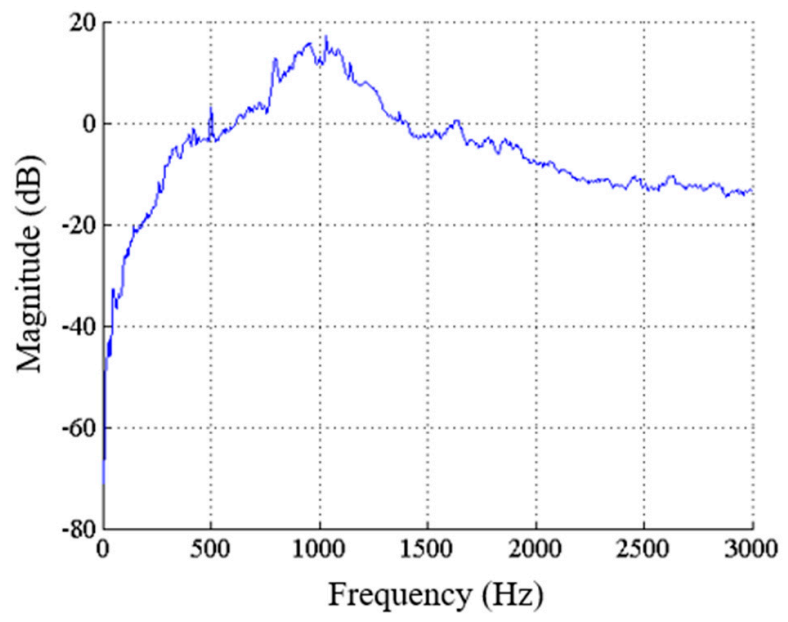

(a)

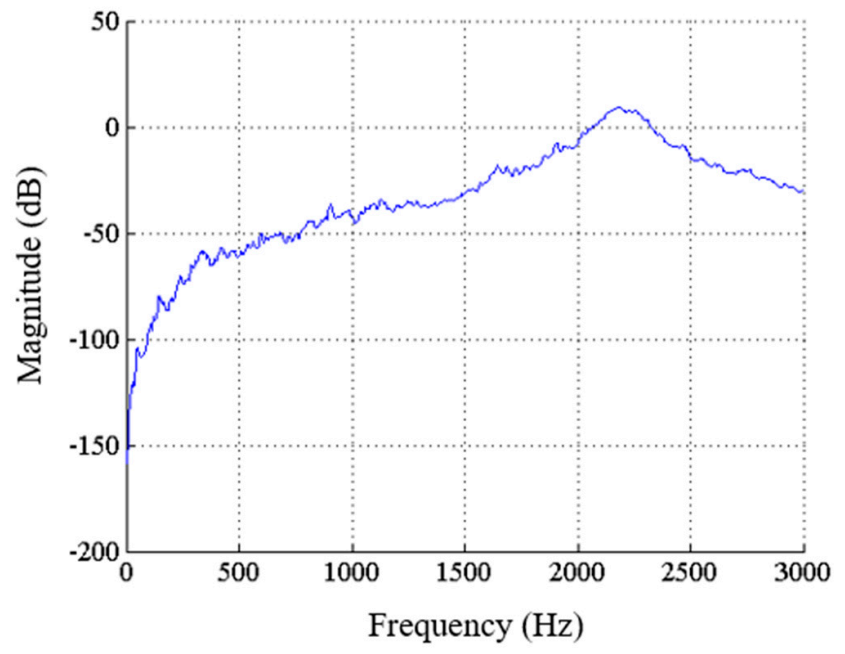

(b)

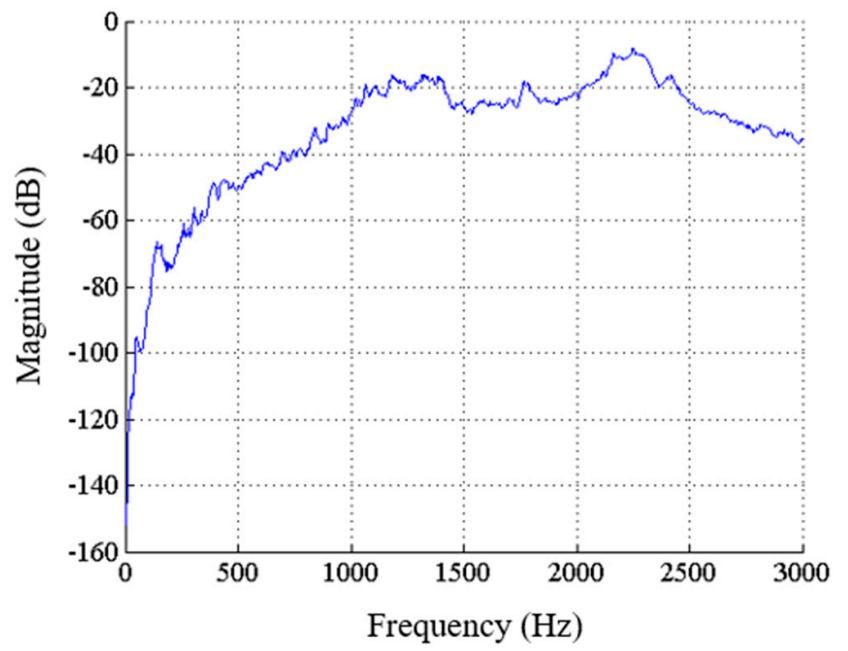

(c)

Figure 7. Frequency response functions for refrigerant flow noise: (a) Slug Flow, (b) Churn Flow, (c) Annular Flow. 
Therefore, refrigerant flow noise which differently occurs in three time intervals could be classified if the refrigerant noise level in the range of $800-2385 \mathrm{~Hz}$ is used. In other words, the dominant frequency band would be observed at $800-1200 \mathrm{~Hz}$ and $100-2300 \mathrm{~Hz}$ in the slug flow noise and churn flow noise, respectively, and at $1056-1356 \mathrm{~Hz}$ and $2085-2385 \mathrm{~Hz}$ in annular noise. In the next section, these frequency characteristics were used as feature vectors for SVM to classify the noises.

\section{Classification of Refrigerant Flow Noise Using Support Vector Machine}

To improve the performance of the SVM classification, kernel functions, which can classify refrigerant flow noise without too much calculation, despite high-dimensional mapping, can be a good option. Therefore, this study used the most widely used RBF kernel.

The training data targeted to apply the classification of refrigerant noise to the SVM are shown in Table 3. Sound level in frequency refers to feature vectors of input data. The sounds can be classified into slug, churn and annular flow noise depending on SPL (dB) in each frequency.

Table 3. Training data for support vector machine.

\begin{tabular}{ccccccc}
\hline \multirow{2}{*}{ Time (s) } & \multicolumn{9}{c}{ Sound Level in Frequency } & \multirow{2}{*}{ Class } \\
\cline { 2 - 6 } & $\mathbf{8 0 0 \mathbf { H z }}$ & $\cdots$ & $\mathbf{8 0 2} \mathbf{~ H z}$ & $\cdots$ & $\mathbf{2 3 8 5} \mathbf{~ H z}$ & \\
\hline 144 & 24.52 & $\ldots$ & 26.00 & $\ldots$ & 20.30 & Slug \\
$\vdots$ & $\vdots$ & & $\vdots$ & & $\vdots$ & $\vdots$ \\
170 & 24.02 & $\ldots$ & 25.80 & $\ldots$ & 20.20 & Slug \\
198 & 20.34 & $\ldots$ & 20.33 & $\ldots$ & 20.10 & Churn \\
$\vdots$ & $\vdots$ & & $\vdots$ & & $\vdots$ & $\vdots$ \\
232 & 20.30 & $\ldots$ & 20.43 & $\ldots$ & 20.12 & Churn \\
313 & 18.34 & $\ldots$ & 18.40 & $\ldots$ & 25.60 & Annular \\
$\vdots$ & $\vdots$ & & $\vdots$ & & $\vdots$ & $\vdots$ \\
317 & 18.40 & $\ldots$ & 18.30 & $\ldots$ & 25.67 & Annular \\
\hline
\end{tabular}

The results of the SVM-based classification are presented in Table 4. The feature vectors are the column of Table 3 . The FT overlap value is $85 \%$, FT block size is 1024 and maximum frequency is about $6100 \mathrm{~Hz}$ (sampling frequency is 12,200 Hz) with ArtemiS SUITE software.

Table 4. Results from classification of refrigerant flow noise by kernel.

\begin{tabular}{lccccc}
\hline & \multicolumn{4}{c}{ Predicted Noise } & \multirow{2}{*}{ Accuracy (\%) } \\
\cline { 2 - 5 } & & Slug & Churn & Annular & \\
\hline \multirow{3}{*}{ Actual Noise } & Slug & 456 & 29 & 15 & 91 \\
& Churn & 12 & 660 & 35 & 93 \\
& Annular & 11 & 13 & 225 & 90 \\
\hline
\end{tabular}

The SVM analysis was performed after selecting parameters within a limited range, and those with the maximum classification performance were searched. For the SVM-based classification, $k$-fold cross validation was used.

The classification accuracy of slug, churn and annular flow noise were 91\%, 93\% and $90 \%$, respectively. Here, classification accuracy was high in churn because the CWT coefficient at the start and end points of this time intervals was high, and refrigerant noise was clear after the significant fluctuation of signals (Figure 3). Likewise, the CWT coefficient at the start and end of annular noise was the lowest because refrigerant noise was lower than other noises in terms of classification accuracy. Further, the SVM analysis was performed after 4-, 6-, 7- and 8-fold cross-validation in addition to 5-fold cross-validation (Table 5). 
Table 5. SVM classification by $k$-fold cross-validation.

\begin{tabular}{cc}
\hline $\boldsymbol{k}$-Fold Cross-Validation & SVM Classification (\%) \\
\hline$k=4$ & 91 \\
$k=5$ & 92 \\
$k=6$ & 92 \\
$k=7$ & 92 \\
$k=8$ & 92 \\
\hline
\end{tabular}

When $k$ was $4,5,6,7$ or 8 , the SVM classification accuracy was almost the same. In particular, it was all the same when $k$ was $\geq 5$. Therefore, this study used 5 -fold cross-validation at the classification of refrigerant flow noise with the SVM.

To increase the classification accuracy of refrigerant flow noise, it was estimated by increasing the number of SVM feature vectors. The block size of the Fourier transform was increased to increase the number of feature vectors. Then, the estimation was compared to the results of the classification using the SVM without applying input data to the kernel (Table 6).

Table 6. Results of classification by SVM and kernel SVM.

\begin{tabular}{ccccc}
\hline \multirow{2}{*}{ Num. of Feature Vectors } & SVM Classification (\%) & Kernel SVM Classification (\%) & \multicolumn{2}{c}{ RBF Kernel Hyperparameter } \\
\cline { 3 - 5 } & & & 90 & 128 \\
\hline 156 & 80 & 92 & 64 & 0.128 \\
318 & 87 & 94 & 64 & 0.128 \\
635 & 92 & 97 & 64 & 0.128 \\
1272 & 92 & 97 & 64 & 0.128 \\
2544 & & & 0.128 \\
\hline
\end{tabular}

In terms of classification accuracy, the kernel method is more accurate. The classification accuracy increases as the number of feature vectors rises. In addition, if the number of feature vectors increases, frequency resolution declines so that each refrigerant flow noise's frequency characteristics are well detected. Therefore, classification accuracy was constant at 1272 or higher in the number of feature vectors, which means that the three time intervals of refrigerant flow noise can be properly classified. After all, classification accuracy is $97 \%$ in the refrigerant flow noises. Then, kernels $C$ and $\delta$ are 64 and 0.128 , respectively.

\section{Verification with Modified Noises}

\subsection{Modified Noise}

Modified refrigerant flow noises were produced for the slug, churn and annular flow noise and the classification performance was compared using the jury test and the SVM. Because the occurrence of refrigerant noises is difficult to predict, it is difficult to generate and evaluate the real refrigerant flow noises. Therefore, verification was performed through the modification of the sources.

The main frequency band level of the Refrigerant noises was increased/decreased by $3 \mathrm{~dB}$ using Sound Forge commercial software. In addition, the frequencies of refrigerant flow noises were shifted. Seven cases of refrigerant noise modified sound were produced from $-9 d B$ to $+9 d B$, and five cases of refrigerant noise were generated to determine the effect of modulation of the frequency band (Tables 7-9). 
Table 7. Fluctuation strength of modified slug noises.

\begin{tabular}{cccc}
\hline Modified Noise & Fluctuation Strength (vacil) & Roughness (asper) & Tonality (tu) \\
\hline Modified Noises $(-9 \mathrm{~dB})$ & 0.0083 & 0.125 & 0.059 \\
Modified Noises $(-6 \mathrm{~dB})$ & 0.0090 & 0.127 & 0.060 \\
Modified Noises $(-3 \mathrm{~dB})$ & 0.0095 & 0.130 & 0.061 \\
Modified Noises $(0 \mathrm{~dB})$ & 0.0098 & 0.134 & 0.063 \\
Modified Noises $(+3 \mathrm{~dB})$ & 0.0097 & 0.137 & 0.065 \\
Modified Noises $(+6 \mathrm{~dB})$ & 0.0104 & 0.139 & 0.066 \\
Modified Noises $(+9 \mathrm{~dB})$ & 0.0112 & 0.140 & 0.067 \\
Modified Noise (Frequency $\times 0.8)$ & 0.0095 & 0.130 & 0.057 \\
Modified Noise (Frequency $\times 0.9)$ & 0.0105 & 0.133 & 0.057 \\
Modified Noise (Frequency $\times 1.0)$ & 0.0098 & 0.134 & 0.063 \\
Modified Noise (Frequency $\times 1.1)$ & 0.0099 & 0.135 & 0.056 \\
Modified Noise (Frequency $\times 1.2)$ & 0.0091 & 0.134 & 0.58 \\
\hline
\end{tabular}

Table 8. Roughness of modified churn noises.

\begin{tabular}{cccc}
\hline Modified Noise & Roughness (asper) & Fluctuation Strength (vacil) & Tonality (tu) \\
\hline Modified Noises $(-9 \mathrm{~dB})$ & 0 & 0.0044 & 0.0041 \\
Modified Noises $(-6 \mathrm{~dB})$ & 0 & 0.0046 & 0.0014 \\
Modified Noises $(-3 \mathrm{~dB})$ & 0.0335 & 0.0047 & 0.0334 \\
Modified Noises $(0 \mathrm{~dB})$ & 0.1130 & 0.0050 & 0.0341 \\
Modified Noises $(+3 \mathrm{~dB})$ & 0.1730 & 0.0051 & 0.0346 \\
Modified Noises $(+6 \mathrm{~dB})$ & 0.2020 & 0.0052 & 0.0356 \\
Modified Noises $(+9 \mathrm{~dB})$ & 0.2362 & 0.0053 & 0.0358 \\
Modified Noise (Frequency $\times 0.8)$ & 0.0397 & 0.0054 & 0.0401 \\
Modified Noise (Frequency $\times 0.9)$ & 0.0903 & 0.0056 & 0.0381 \\
Modified Noise (Frequency $\times 1.0)$ & 0.1130 & 0.0050 & 0.0341 \\
Modified Noise (Frequency $\times 1.1)$ & 0.0980 & 0.0057 & 0.0337 \\
Modified Noise (Frequency $\times 1.2)$ & 0.0721 & 0.0056 & 0.0334 \\
\hline
\end{tabular}

Table 9. Tonality of modified annular noises.

\begin{tabular}{cccc}
\hline Modified Noise & Tonality (tu) & Fluctuation Strength (vacil) & Roughness (asper) \\
\hline Modified Noises $(-9 \mathrm{~dB})$ & 0.0484 & 0.0657 & 0.509 \\
Modified Noises $(-6 \mathrm{~dB})$ & 0.0528 & 0.0636 & 0.511 \\
Modified Noises $(-3 \mathrm{~dB})$ & 0.0568 & 0.0621 & 0.521 \\
Modified Noises $(0 \mathrm{~dB})$ & 0.0618 & 0.0608 & 0.530 \\
Modified Noises $(+3 \mathrm{~dB})$ & 0.0696 & 0.0584 & 0.541 \\
Modified Noises $(+6 \mathrm{~dB})$ & 0.0734 & 0.0575 & 0.545 \\
Modified Noises $(+9 \mathrm{~dB})$ & 0.0779 & 0.0585 & 0.547 \\
Modified Noise (Frequency $\times 0.8)$ & 0.0675 & 0.0557 & 0.546 \\
Modified Noise (Frequency $\times 0.9)$ & 0.0634 & 0.0609 & 0.547 \\
Modified Noise (Frequency $\times 1.0)$ & 0.0618 & 0.0608 & 0.532 \\
Modified Noise (Frequency $\times 1.1)$ & 0.0644 & 0.0590 & 0.530 \\
Modified Noise (Frequency $\times 1.2)$ & 0.0559 & 0.0591 & 0.541 \\
\hline
\end{tabular}

The modified noises of the slug flow were produced by changes in the amplitude and frequency of the signal for $800-1200 \mathrm{~Hz}$. It is assumed that the slug flow noise can be expressed as fluctuation strength because the value of fluctuation strength changes a lot compared to the other two (Table 7). The fluctuation strength was used to determine whether the modified noises were characterized as the slug noise.

Fluctuation strength is used to quantify subjective perception of amplitude modulation of a sound. The unit of measure for fluctuation strength is the vacil. One vacil is defined as the fluctuation strength produced by a $1000 \mathrm{~Hz}$ tone of $60 \mathrm{~dB}$ which is $100 \%$ amplitude modulated at $4 \mathrm{~Hz}$ [14]. The following equation shows the variation of fluctuation strength $(F)$ with masking depth $(\Delta L)$, and modulation frequency $\left(f_{\bmod }\right)$ :

$$
F(z)=\frac{0.032 \cdot \Delta L(z) \cdot \Delta z}{f_{\text {mod }}(z) / 4+4 / f_{\bmod }(z)}
$$


where $z$ denotes critical band rate [20].

The modified noises of the churn flow were produced by changes in the amplitude and frequency of the signal for $2100-2300 \mathrm{~Hz}$. The roughness was used to determine whether the modified noises were characterized as the churn noise because the value of roughness changes a lot compared to other values (Table 8). Roughness is one of the Zwicker parameters, representing the subjective perception of rapid amplitude modulation of a sound. The unit of measure for roughness is the asper. One asper is defined as the roughness produced by a $1000 \mathrm{~Hz}$ tone of $60 \mathrm{~dB}$ which is $100 \%$ amplitude modulated at $70 \mathrm{~Hz}$ [20].

$$
R(z)=0.0003 \cdot f_{\bmod }(z) \cdot \Delta L_{E}(z) \cdot \Delta z
$$

where $f_{\text {mod }}$ is the frequency of modulation and $\Delta L_{\mathrm{E}}$ is the perceived masking depth and $z$ denotes critical band rate.

The modified noises of the annular flow were produced by changes in the amplitude and frequency of the signal for $1056-1356 \mathrm{~Hz}$ and $2085-2385 \mathrm{~Hz}$. It is assumed that the annular flow noise can be expressed as tonality because the value of tonality changes a lot compared to the other two (Table 9). Therefore, the tonality was used to determine whether the modified noises were characterized as the annular noise.

The equation of tonality is as follows:

$$
\begin{gathered}
t_{b}=-0.4343 \log \left(\frac{\sqrt{[r \cos \theta-\hat{r} \cos \hat{\theta}]^{2}+[r \sin \theta-\hat{r} \sin \hat{\theta}]^{2}}}{r+\hat{r}}\right)-0.30103 \\
\hat{r}(t, \omega)=r(t-1, \omega)+\{r(t-1, \omega)-r(t-2, \omega)\} \\
\hat{\theta}(t, \omega)=\theta(t-1, \omega)+\{\theta(t-1, \omega)-\theta(t-2, \omega)\}
\end{gathered}
$$

where $r$ denotes magnitude at time $t$ and frequency $\omega$ and $\theta$ is phase at $t$ and frequency $\omega$ [21].

Modified noises were generated based on slug, churn, annular and any interval where no refrigerant noise occurred. A total of 48 sound sources was generated.

All three kinds of modified sounds show that the corresponding Zwicker parameter changes. Expert assessments and classification using SVMs were carried out for these modified sources.

In order to conduct a jury test of the modified sounds, the experimenters were selected as experts of refrigerant noises. In general, air conditioning refrigerant noises are low sound level and vary depending on the flow pattern. In addition, it is necessary to conduct a jury test using experts who have experience because it is difficult to detect changes. The jury test was conducted using headphones. Headphones were used to allow all experts to conduct the jury test in the same environment because speakers could distort the sound depending on the location and distance. The evaluation was conducted after playing for $10 \mathrm{~s}$ for each sound source so that the sound could be judged sufficiently. The rating scale method was used for the jury test. This is because an evaluation method that is convenient for the listener is needed. The rating scale method is a common method of data collection that is used to gather comparative information about a specific research subject. Specifically, a rating scale is a type of multiple-choice question that allows survey respondents to assign a value to a product or service. In this study, three scales were used, and the expert listeners were asked to answer whether they could hear or not, or they could select a neutral opinion (not sure).

In addition, in order to take into account the anchor effect, the lowest and highest levels of sound were played first to set the standard. This is because the judgment of a stimulus, along with its own characteristics, is influenced and changed according to the order in which it is placed. Finally, two repeated jury evaluations were conducted to obtain reliable evaluation results, and more reliable results were obtained through correlation analysis between the results of the same hearing evaluation results. 


\subsection{Comparison of Results between Jury Test and SVM}

The expert listeners were asked whether they could hear or not, or if they had a neutral opinion (not sure). The results are shown in Table 10. To summarize the results, the results of the jury test showed more than $95 \%$ classification accuracy for three types of noise. The classification accuracy was over $90 \%$ when classified using the pre-learned hyperplane through SVM. The results of the jury test and kernel SVM showed that the lower the $\mathrm{dB}$ of the noise source produced in general, the greater the error. This means that the certain level of refrigerant noise was masked by other noises. To plot the classification performance of the kernel SVM, two arbitrary axes were selected to represent the distribution and classification of the signal (Figure 8). This is a two-dimensional representation of 1272 dimensions (the number of characteristic vectors) by selecting two arbitrary axes.

Table 10. Results of classification by kernel SVM and jury test.

\begin{tabular}{ccc}
\hline Class & Kernel SVM Classification (\%) & Jury Test (\%) \\
\hline Slug flow & $92 \%$ & $98 \%$ \\
Churn flow & $94 \%$ & $95 \%$ \\
Annular & $90 \%$ & $95 \%$ \\
\hline
\end{tabular}

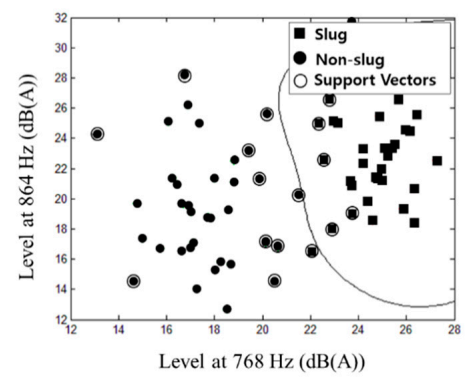

(a)

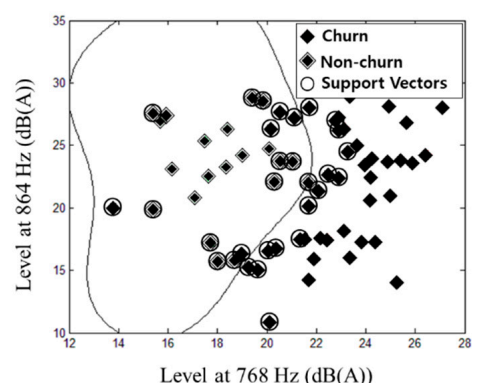

(b)

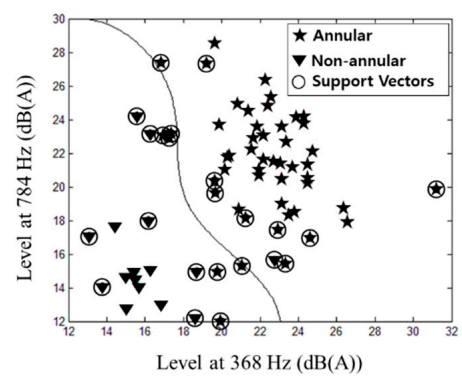

(c)

Figure 8. Kernel SVM classification for modified noises (2-D plot): (a) Slug flow, (b) Churn flow, (c) Annular flow. 
The accuracy of the jury test was higher than that of the kernel SVM (Table 10). This is because the learning data and test data are different. If the data sets are similar, the accuracy is increased, as shown in Table 6 in the previous section. The performance of the kernel SVM may be somewhat reduced because the test data changed as the noise was modified. However, it shows a high accuracy of more than $90 \%$.

\section{Conclusions}

The present study proposed a method that uses the CWT and the SVM to classify the refrigerant flow noise generated in air conditioners. The air conditioner refrigerant flow noise was identified as an abnormal signal, the first- and second-order moments of which varied with time. The start and end points of refrigerant flow noise generation were located using CWT coefficients, and a sound listening test revealed that different levels of noise were generated in three time intervals. Further, a CWT-based analysis of time-frequency characteristics revealed that the refrigerant flow noise exhibited different frequency characteristics in three different time intervals. The identified time-frequency characteristics were selected as the SVM's feature vectors. Finally, kernel hyperparameters were determined by a grid search method and the findings of the SVM analysis with 5-fold cross-validation showed that refrigerant flow noise could be classified with an accuracy of $92 \%$. Noise sources were modified and classification verification was performed, indicating that this proposed method could be used for noise classification.

Funding: This research was funded by Honam University in 2019, grant number 2019-0104.

Conflicts of Interest: The author declares no conflict of interest.

\section{References}

1. Kandlikar, S.G. Two-phase flow patterns, pressure drop, and heat transfer during boiling in minichannel flow passages of compact evaporators. Heat Transf. Eng. 2002, 23, 5-23. [CrossRef]

2. Chen, L.; Tian, Y.S.; Karayiannis, T.G. The effect of tube diameter on vertical two-phase flow regimes in small tubes. Int. J. Heat Mass Transf. 2006, 49, 4220-4230. [CrossRef]

3. Han, H.S.; Jeong, W.B.; Kim, M.S.; Lee, S.Y.; Seo, M.Y. Reduction of the refrigerant-induced noise from the evaporator-inlet pipe in a refrigerator. Int. J. Refrig. 2010, 33, 1478-1488. [CrossRef]

4. Han, H.S.; Jeong, W.B.; Kim, M.S.; Kim, T.H. Analysis of the root causes of refrigerant-induced noise in refrigerators. J. Mech. Sci. Technol. 2009, 23, 3245-3256. [CrossRef]

5. Crocker, M.J.; Arenas, J.P.; Dyamannavar, R.E. Identification of noise sources on a residential split-system air-conditioner using sound intensity measurements. Appl. Acoust. 2004, 65, 545-558. [CrossRef]

6. Chen, C.L.J.J.P; Chen, Z.J.; Tian, J.; OuYang, H.; Du, Z.H. Experimental and numerical study on aeroacoustic sound of axial flow fan in room air conditioner. Appl. Acoust. 2007, 684, 458-472.

7. Soeta, Y.; Shimokura, R. Sound quality evaluation of air-conditioner noise based on factors of the autocorrelation function. Appl. Acoust. 2017, 124, 11-19. [CrossRef]

8. Susini, P.; McAdams, S.; Winsberg, S.; Perry, I.; Vieilard, S.; Rodet, X. Characterizing the sound quality of air-conditioning noise. Appl. Acoust. 2004, 65, 763-790. [CrossRef]

9. Jianxing, T.; Houming, D.; Shengmei, Y. Development and Application of Techniques for Evaluating Sound Quality of Air-conditioners. J. Vib. Meas. Diagn. 2001, 3, 214-219.

10. Jeong, U.C.; Kim, J.S.; Jeong, J.E.; Yang, I.H.; Oh, J.E. Development of a sound quality index for the wash cycle process of front-loading washing machines considering the impacts of individual noise sources. Appl. Acoust. 2015, 87, 183-189. [CrossRef]

11. Chen, B.; Kang, Y.P.; Li, P.Y.; Xie, W.P. Detection on structural sudden damage using continuous wavelet transform and lipschitz exponent. Shock Vib. 2015, 2015, 832738. [CrossRef]

12. $\mathrm{Da} \mathrm{Wu}, \mathrm{J} . ; \mathrm{Chen}, \mathrm{J} . \mathrm{C}$. Continuous wavelet transform technique for fault signal diagnosis of internal combustion engines. NDT E Int. 2006, 39, 304-311. 
13. Saha, S.; Mukherjee, D.; Mukhopadhyay, S. Online detection and location estimation of earthquake events using continuous wavelet transform. In Proceedings of the 2016 IEEE First International Conference on Control, Measurement and Instrumentation (CMI), Kolkata, India, 8-10 January 2016; pp. 77-82.

14. Nenadic, Z.; Burdick, J.W. Spike detection using the continuous wavelet transform. IEEE Trans. Biomed. Eng. 2005, 52, 74-87. [CrossRef] [PubMed]

15. Zhang, Y.; Guo, Z.; Wang, W.; He, S.; Lee, T.; Loew, M. A comparison of the wavelet and short-time fourier transforms for Doppler spectral analysis. Med. Eng. Phys. 2003, 25, 547-557. [CrossRef]

16. Demir, B.; Bruzzone, L. Fast and accurate image classification with histogram based features and additive kernel SVM. In Proceedings of the 2015 IEEE International Geoscience and Remote Sensing Symposium (IGARSS), Milan, Italy, 26-31 July 2015; pp. 2350-2353.

17. Zhu, S.; Xu, C.; Wang, J.; Xiao, Y.; Ma, F. Research and application of combined kernel SVM in dynamic voiceprint password authentication system. In Proceedings of the 2017 IEEE 9th International Conference on Communication Software and Networks (ICCSN), Guangzhou, China, 6-8 May 2017; pp. 1052-1055.

18. Grossmann, A.; Kronland-Martinet, R.; Morlet, J. Reading and Understanding Continuous Wavelet Transforms. In Wavelts; Springer: Berlin/Heidelberg, Germany, 1989; pp. 2-20.

19. Manning, C.D.; Raghavan, P.; Schutze, H. Introduction to Information Retrieval; Cambridge University Press: Cambridge, UK, 2008.

20. Zwicker, E.; Fastl, H. Psychoacoustics: Facts and Models, 3rd ed.; Springer: Berlin/Heidelberg, Germany, 2007.

21. Thjayaraman, J.; Spanias, A. Analysis of the MPEG-1 Layer III (MP3) Algorithm Using MATLAB; Morgan \& Claypool: San Rafael, CA, USA, 2011.

(C) 2020 by the author. Licensee MDPI, Basel, Switzerland. This article is an open access article distributed under the terms and conditions of the Creative Commons Attribution (CC BY) license (http://creativecommons.org/licenses/by/4.0/). 